\title{
Intermédialités
}

Histoire et théorie des arts, des lettres et des techniques

Intermediality

History and Theory of the Arts, Literature and Technologies

\section{Introduction. Le multiple et le transmissible}

\section{Suzanne Paquet}

Numéro 17, printemps 2011

reproduire

reproducing

URI : https://id.erudit.org/iderudit/1005745ar

DOI : https://doi.org/10.7202/1005745ar

Aller au sommaire du numéro

Éditeur(s)

Revue intermédialités (Presses de l’Université de Montréal)

ISSN

1705-8546 (imprimé)

1920-3136 (numérique)

Découvrir la revue

Citer ce document

Paquet, S. (2011). Introduction. Le multiple et le transmissible. Intermédialités /

Intermediality, (17), 9-17. https://doi.org/10.7202/1005745ar 


\title{
Introduction \\ Le multiple et le transmissible*
}

\author{
Suzanne Paquet
}

a question de la reproduction des images, du son, des œuvres d'art se trouve au cour de l'actualité, mais elle n'est certes pas nouvelle. De la représentation par des moyens manuels jusqu'à l'encodage numérique, il y eut une longue série de passages par des médias de plus en plus mécanisés, des technologies de plus en plus complexes. Ces avancées constantes indiquent à quel point le désir de reproduire, qui est désir de faire circuler, a toujours été vif. Et si la problématique de la reproduction est si aiguë aujourd'hui, c'est que tout est voué à être dupliqué, copié, à proliférer pour mieux se propager à travers un large régime, celui du numérique, qui aurait absorbé bien des techniques, rendant tout similaire par le code - depuis le mode d'enregistrement jusqu'à celui de transmission et de diffusion - et infiniment multipliable.

Le souhait de reproduire fut en premier lieu, on le sait, celui d'imitation de la nature. C'est d'abord par des modes plastiques et manuels - dessin, peinture et moulage - que l'on est arrivé à copier les choses du monde. Et lorsqu'il devient possible de reproduire la nature en des œuvres dignes d'admiration, on cherchera à dupliquer celles-ci, d'abord à la main puis par des méthodes autorisant des tirages multiples. Par ailleurs, ce désir d'imitation de la nature fait largement appel à l'idée de paysage qui suscitera des réflexions sur l'espace et le temps. Tout à la fois la chose et son image, perception in situ et représentation, le paysage se

* Certains textes qui paraissent ici ont été généreusement partagés par leurs auteurs (dans des versions parfois différentes) lors de l'atelier international «Photographie, mobilités, intermédialités » tenu à l'Université de Montréal en avril 2011. Il s'agit de ceux de Jan von Brevern, Karla McManus, Ingrid Hölzl et Anne Bénichou; certaines des images de Louise Merzeau y ont aussi été présentées. Nos remerciements vont au Centre de recherche sur l'intermédialité et au Conseil de recherches en sciences humaines du Canada (CRSH) qui ont contribué à la tenue de cet atelier et à l'élaboration de ce numéro Reproduire/Reproducing. 
conçoit comme un médium ${ }^{1}$ qui, s'agrégeant à d'autres médias, peut ainsi devenir transmissible. De même, par le biais de leur reproduction, les œuvres d'art seront rendues mobiles et voyageuses. Les copies et fac-similés d'œuvres d'art, leur mobilité - temporelle tout aussi bien que spatiale -, le paysage, constituent les principales lignes de force suivant lesquelles - sans que cela soit restrictif - ce numéro d'Intermédialités a été conçu.

Reproduire, c'est faire passer un objet, une portion de territoire, une image, une voix, une œuvre, d'une matière à une autre, d'un média à un autre, tout aussi bien que d'un lieu à un autre. C'est également, à bien des égards, vouloir les faire passer d'un temps à un autre. L'acte de reproduction nous fait donc entrer dans de curieuses sphères spatiotemporelles où les lieux et les sites, les œuvres, visuelles ou sonores, les images, fixes ou mouvantes, deviennent ubiquistes, visibles, audibles, perceptibles en différents endroits, en des temps mêmes ou des temps autres, s'hybridant et se croisant avec d'autres médias.

La propagation - La gravure fut l'un des premiers vecteurs importants de la circulation des œuvres d'art. Comme l'explique Stéphane Roy dans «Imiter, reproduire, inventer: techniques de gravure et statut du graveur en France au $18^{\text {e }}$ siècle ", elle fut un art utile, apte à multiplier les œuvres afin de les mettre à la portée des amateurs. La gravure d'interprétation ou de traduction, moyen favori de diffuser les œuvres de la peinture et du dessin à partir du $17^{\mathrm{e}}$ siècle sera toutefois vouée à la disparition avec l'apparition de la photographie au $19^{\mathrm{e}}$ siècle $^{2}$. Gravure d'interprétation et photographie ont en commun le pouvoir de stimuler la «mise en spectacle des arts» et de les transformer en produits de consommation. Elles partagent également le mépris dans lequel bien des artistes les tiennent, les voyant toutes deux comme des applications purement techniques et plutôt vulgaires. Mais, comme le montre Magali Le Mens à partir de l'exemple d'Odilon Redon, dans «Puissance littéraire de la reproduction de l'œuvre d'art » qui traite de la reproduction des œuvres par la photographie, ce ne sont pas tous les artistes qui dédaignent ce médium. Théophile Gauthier ne disait-il pas de la

1. W. J. T. Mitchell, «Imperial Landscape», dans W. J. T. Mitchell (dir.), Landscape and Power, Chicago, University of Chicago Press, 2002, p. 5.

2. En revanche, ce «décès» permettra le renouveau de l'estampe originale. Voir Pierre-Lin Renié, «Guerre commerciale, bataille esthétique : la reproduction des œuvres d'art par l'estampe et la photographie, 1850-188o », dans Véronique Goudinoux et Michel Weemans (dir.), Reproductibilité et irreproductibilité de l'œuvre d'art, Bruxelles, La lettre volée, 2001, p. 78 . 
photographie qu' «elle a vraiment fait merveille, même dans l'excès de son zèle elle a changé en très beaux tableaux des toiles assez médiocres » ?3

Il est incontestable que la photographie assumera, très vite, un rôle majeur dans les techniques de reproduction, évinçant maints procédés précédemment usités. Elle aura notamment contribué à la tombée en désuétude de la pratique de l'estampage, une technique de moulage au papier d'origine chinoise appréciée des explorateurs-archéologues et qui «connut son apogée durant la seconde moitié du $19^{\mathrm{e}}$ siècle avant de disparaître devant les progrès de la photographie et de ses procédés de reproduction ${ }^{4}$ ». Une méthode de prise d'empreinte, mécanisée, en aura chassé une autre, essentiellement manuelle, sans que la perte du relief ne semble gêner quiconque. C'est dire la croyance profonde envers la qualité d'enregistrement mécanique de l'image photographique qui, très tôt, s'installe. Les vues d'optiques - qu'évoque également Stéphane Roy -, ces gravures montrant des panoramas de grandes capitales à travers un ensemble de lentilles et de miroirs produisant des perspectives saisissantes, seront elles aussi éliminées par les «vues enregistrées ${ }^{5}$ » obtenues par la photographie, celles au stéréoscope tout particulièrement. Au même titre que la peinture et les œuvres d'art, les monuments et panoramas, tout comme le paysage, sont en effet des motifs que très tôt l'on a voulu reproduire par la photographie. C'est que «la photographie se voyait assignée dès sa naissance (et notamment par Arago) la conquête de la totalité du monde par sa reproduction automatique. Les tout premiers albums intitulés Excursions daguerriennes, qui rassemblaient des vues de pays plus ou moins éloignés et prises par quelques "médiateurs automatiques" envoyés à la hâte, témoignent de cet immédiat mouvement d'appropriation du monde par sa conversion en images ${ }^{6}$. " Mais il faut néanmoins rappeler que les Excursions daguerriennes, Les vues et monuments les plus remarquables du monde commercialisées par N. P. Lerebours dès 1841, étaient en fait des gravures exécutées à partir de daguerréotypes.

3. Théophile Gauthier, «L'œuvre de Paul Delaroche photographiée», L'Artiste, janvier-avril 1858, p. 153-155, dans André Rouillé, La photographie en France, textes \& controverses. Une anthologie 1816-1871, Paris, Macula, 1989, p. 243.

4. Pascal Monge, «Désiré Charnay. De la photographie à l'archéologie », dans "Le Yucatan est ailleurs". Expéditions photographiques (1857-1886) de Désiré Charnay, Paris, Musée du Quai Branly / Actes Sud, 2007, p. 69.

5. L'expression (recorded sights) est de Joël Snyder, «Territorial photography», dans Mitchell, 2002, p. 175-202.

6. Éric Michaud, «Daguerre, un Prométhée chrétien», Histoire de l'art. Une discipline à ses frontières, Paris, Hazan, 2005, p. 165 (je souligne). 
La topographie et le temps - Le paysage, considéré comme outil ou modalité de représentation du territoire, utilise d'autres médias et se croise avec eux pour former des hybrides ${ }^{7}$. Très vite, paysage et photographie deviennent indissociables avant que de nouveaux hybrides naissent de leur amalgame avec d'autres médias ou véhicules. À bien des égards, le photographique, le reproductible et le géographique travaillent donc de concert. Ainsi, Jan von Brevern montre dans «Fototopografia» comment des scientifiques fascinés par la véracité de la photographie ont tenté, dès les années 186o, de coupler cette technique avec la topographie afin d'extraire aux images photographiques l'information nécessaire pour cartographier des territoires difficiles d'accès. En le photographiant, puis en le transposant d'un procédé à un autre pour le réinterpréter graphiquement, on a voulu faire du paysage, pourtant objet esthétique plutôt que donnée scientifique, l'origine de documents cartographiques utilisables. La même croyance envers l'objectivité et la neutralité mécanique de l'enregistrement photographique qui permit qu'elle supplante les autres types de reproduction d'œuvres d'art, a autorisé ce détournement ou cette instrumentalisation du médium paysage au profit de la carte, modalité de représentation considérée comme une autre forme de reflet de la nature. Le processus de repeat photography ou re-photographie par lequel on duplique, selon une échelle temporelle prédéterminée, une photographie qui elle-même reproduit une parcelle de territoire, est issu de cette même conviction qui veut que la captation automatique soit neutre. Ce phénomène particulier est étudié par Karla McManus dans «Objective Landscapes: The Mediated Evidence of Repeat Photography». Comme c'est le cas dans le Rocky Mountain Repeat Photography Project, les re-photographies se présentent généralement sous forme de séries qui déploient une temporalité séquentielle du site (ou de l'image) reproduit(e), soit une suite ouverte de moments coupés et cadrés pouvant être indéfiniment prolongée. De cette manière, le paysage peut se concevoir comme une série d'instants, une spatialité elle-même inscrite dans un flux temporel.

Au-delà de la seule dimension visuelle, l'espace et le temps peuvent aussi être produits ou structurés par l'audible et le sonore de sorte qu'une collectivité puisse se construire en termes médiaux. Comme l'explique Cornelia Epping-Jäger dans «Hitler's voice: The Loudspeaker under National Socialism», la «voix»

7. L'idée de «l'hybridation ou de la compénétration des médias» est proposée par Marshall McLuhan. Voir, entre autres: «L'énergie hybride» et «La photographie. Le bordel imaginaire», dans Pour comprendre les médias. Les prolongements technologiques de l'homme [1964], Montréal, Bibliothèque québécoise, 2001, p. 95-105 et p. 295-315. 
d'Hitler et, plus largement, celle du régime national-socialiste, fut modulée et amplifiée par des technologies acoustiques d'une efficacité toujours surprenante aujourd'hui, avant de devenir omniprésente en Allemagne. Des performances vocales «de masse» données par de multiples orateurs pendant la montée en force du parti jusqu'aux radiodiffusions de la voix du Führer après son arrivée au pouvoir, cette ubiquité de la voix grâce aux moyens techniques employés aurait contribué à restructurer l'espace. Elle aurait ainsi remodelé l'espace politique et communicationnel de l'époque, qualifié ici de «media-political landscape».

La photographie omniprésente - Dans «Le martyre de sainte Sébastienne. Retour sur une vision de Victor Burgin », Vincent Bruyère met peinture et photographie en relation réflexive en examinant l'image d'un tableau imaginaire retournée par un miroir, lui-même photographié. En poursuivant le spectre de Sébastienne à travers ses diverses incarnations chez Helmut Newton, Louise Bourgeois et Antonello de Messine, il est ici question d'une insistance spécifique, «c'est-à-dire du retour sur l'image et du retour du regard dans l'image, mais également de ce qui est en jeu dans l'opérativité du préfixe re-». Et dans ces déplacements - ces effets de reflet et de retour - le photographique semble continuellement présent.

La photographie se pose donc, dès son avènement, en mode tyrannique de reproduction du monde aussi bien que des œuvres de l'art. Seule l'image numérique qui, pour bien des auteurs, ne serait plus de l'ordre de la photographie mais participerait plutôt d'un « régime général de l'image ${ }^{8}$ » aura réussi à l'évincer. Dans ce régime toutefois, si l'on suit l'analyse proposée par Ingrid Hölzl dans «The Photographic-Now: David Claerbout's Vietnam» à propos d'un travail de vidéaste réutilisant la célèbre photographie de presse d'un avion américain abattu par sa propre artillerie, les catégories duelles image fixe ou en mouvement, image imprimée ou projetée, enregistrée ou conçue par le calcul, deviendraient caduques puisque le code, désormais unique, rend incertaine toute différenciation. L'auteure interroge, à travers l'image devenue signal, à la fois un état présent (un maintenant) de la photographie et la nouvelle relation de celle-ci au présent qui serait dépassement de cet état d'immédiatement différé caractéristique de la photographie argentique. Il y aurait comme une perpétuation - une reproduction - infinie ou indéfinie de l'instant présent, correspondant vraisemblablement au temps vécu aujourd'hui.

8. Voir, par exemple: Quentin Bajac, Après la photographie? De l'argentique à la révolution numérique, Paris, Gallimard, coll. «Découvertes Gallimard», 2010, p. 119. 
En considérant la multiplication et le foisonnement des lieux et des modalités de présence d'une artiste et de ses œuvres, Anne Bénichou fait état d'un cas tout à fait singulier. Son étude "Marina Abramović: The Artist Is [Tele]Present » montre en effet comment, dans le cas des performances de cette artiste, les médias et leurs interactions, les remédiations s'additionnant, se répondant et se multipliant, une œuvre d'art peut ouvertement se décliner en divers supports, occurrences ou incarnations, successives ou concurrentes. Par cette multiplication intermédiale des formes de l'œuvre, d'actions ponctuelles en documents et en re-performances, de l'archive au musée en passant par le Web, les habituelles positions relatives à l'original et à la reproduction se voient ici remises en question, l'artiste décidant de la légitimité de chacune des occurrences en y distribuant, pour ainsi dire, l'aura.

L'aura, toujours - La migration de la photographie vers le numérique ne serait pas le moindre des déplacements: il pourrait correspondre à un glissement, un essoufflement généralisé de la prééminence de l'original. Le numérique a en effet cette singularité de n'être pas singulier, mais multipliable à l'infini, remarquablement transmissible et partageable. Si, avec l'image numérique, il reste quelque chose de la nécessité d'une contiguïté physique avec le référent premier, les reproductions portent non seulement avec elles la menace de perte d'unicité propre à la photographie qu'évoquait Walter Benjamin, mais elles sont désormais parfaitement identiques à la copie zéro - sans perte de génération entre la captation originaire et ses multiples. Dans ce régime, la reproduction d'une œuvre pourrait-elle devenir parfaitement équivalente à l'œuvre originale? L’œuvre d'art, prenant en quelque sorte la place du négatif de la photographie, se ferait alors l'unique «instrument de production des objets multiples ${ }^{9}$ » voués à être disséminés. Elle correspondrait, comme Bruno Latour et Adam Lowe le suggèrent dans «La migration de l'aura, ou comment étudier un original par le biais de ses fac-similés ", à une matrice - ce qu'était le négatif argentique selon Genette - dont on prélèverait l'empreinte aux fins de réactualiser l'œuvre ailleurs. En termes temporels, il y aurait là anachronisme: «le processus d'empreinte est-il contact de l'origine ou bien perte de l'origine? Manifestet-il l'authenticité de la présence (comme processus de contact) ou bien, au contraire, la perte d'unicité qu'entraîne sa possibilité de reproduction? Produit-il

9. Gérard Genette, L'œeuvre de l'art. Immanence et transcendance, Paris, Seuil, 1994, p. 53,59 . 
l'unique ou le disséminé? L'auratique ou le sériel ${ }^{10}$ ? 》 Dans le cas particulier des Noces de Cana de Véronèse dont parlent Latour et Lowe, l'œuvre reproduite l'a été par l'intermédiaire d'une numérisation par captation plutôt que par contact, ce qui n'empêche pas, selon les auteurs, son aura de se déplacer de la matrice vers son fac-similé, lui-même replacé dans le lieu originel palladien de San Giorgio à Venise. Dans sa réponse à ce texte quelque peu provocateur, Johanne Lamoureux met non seulement en cause la possibilité que l'aura d'une œuvre puisse être voyageuse, mais elle fait également remarquer que les déplacements physiques que l'on (a) fait subir aux œuvres originales - et ce sont bien des changements de lieux qui occasionnent ces réflexions autour de l'aura - révèlent les possibles usages politiques de la duplication numérique. À leur tour, ceux-ci mettent en avant la question difficile et toujours d'actualité du déplacement et de la restitution des œuvres.

La démultiplication des images, de leurs lieux d'élection et de leurs temps d'existence, est aujourd'hui devenue chose courante. La communication électronique et le numérique concourent évidemment à organiser et à faire percevoir autrement l'espace et le temps; et la possibilité toujours accrue de tout reproduire participe assurément de ces transformations spatiotemporelles. Comme l'affirmait Marc Augé, la diversité spatiale, désormais portée par les nouvelles technologies et leur (non-)lieu d'évolution qu'est le cyberespace, absorberait la diversité temporelle ${ }^{11}$ puisque tout devient équivalent et accessible dans l'instant. Non sans risque d'égarement, telles ces images orphelines que Louise Merzeau s'emploie à sauvegarder par montage numérique dans la série qu'elle leur consacre. Merzeau fait ici œuvre, en s'appropriant et en faisant réapparaître ailleurs, sans toutefois qu'elles ne disparaissent de leurs séjours précédents, des photographies égarées ou abandonnées errant dans le cyberespace, réitérant ainsi leur effet de présence et leur offrant une nouvelle vie.

Ce numéro Reproduire/Reproducing s'est ouvert, et se referme, sur le papier, un médium tout aussi essentiel que souvent oublié. Comme le rappelle en guise d'entrée en matière Lothar Müller dans «L’imprimé et le non-imprimé. Théorie

10. Georges Didi-Huberman, La ressemblance par contact. Archéologie, anachronisme et modernité de l'empreinte, Paris, Les Éditions de Minuit, coll. «Paradoxe», 2008, p. 18.

11. Marc Augé, Pour une anthropologie de la mobilité, Paris, Payot \& Rivages, 2009, p. 57 . 
des médias et poétique du papier ", ce dernier est apparu bien avant l'imprimerie et, trop souvent seulement considéré comme le «carburant» de la presse à imprimer, continue d'exister indépendamment de celle-ci dans la «Galaxie Gutenberg», contribuant, en retour, à fortement valoriser le manuscrit. Le papier, dont on veut nous faire croire aujourd'hui qu'il n'est plus nécessaire, l'écran et les techniques numériques se substituant à ce médium, fut et reste malgré tout un véhicule essentiel de transmission des écrits, des idées, des images: il suffit de considérer tout ce qui, par le papier, sur papier, en papier, se conserve et peut ainsi circuler.

C'est aussi pourquoi ce dossier se clôt sur un document d'archive qui retrace l'apparition et la destinée du papier à travers l'histoire des cultures mondiales. Il s'agit d'un extrait inédit de «The Coming of Paper», chapitre du dernier grand projet inachevé d'Harold Innis (1894-1952), A History of Communications - et dont la publication s'inscrit dans une visée de documentation d'une archéologie de la pensée intermédiale inaugurée, dans notre numéro 12, avec le texte de Paul Zumthor sur l'oralité. C'est comme historien de l'économie qu'Innis s'intéressa en pionnier à l'histoire des communications en analysant les relations entre les formes médiatiques dominantes et celles de pouvoir et de contrôle social ${ }^{12}$. On sait combien, en conjonction avec les travaux d'Eric Havelock, Edmund Carpenter et Marshall McLuhan, ses théories ont contribué à la constitution de la dite École de communication de Toronto dont il peut être considéré comme le spiritus rector. Consacré à l'émergence du papier, cet extrait du chapitre IV des quelque 1400 pages du manuscrit de A History of communications esquisse l'histoire culturelle d'un médium dont l'apparition remonte à la Chine du début de notre ère, et dont la diffusion suit les routes d'expansion des religions mondiales, en particulier le bouddhisme et l'islam, avant d'atteindre l'Europe au cours du Moyen Âge. S'intéressant parallèlement aux techniques de production d'encre, au rôle de la xylographie et à l'introduction des caractères mobiles, Innis y pointe quel effet ce formidable rival du parchemin eut sur les évolutions les plus significatives de l'Occident telles que le développement du crédit, la renaissance de l'Antiquité, la Réforme et l'émergence de l'État moderne.

Cet inédit n'existait jusqu'ici qu'en tant que document dactylographié sur papier - et sous la forme de quelques exemplaires de microfiches -, autre preuve, s'il en faut, que le papier reçoit, transporte et transmet les écrits, sans l'aide de

12. Voir, en particulier: Empire and Communications, Oxford, Clarendon Press, 1950 et The Bias of Communication, Toronto, University of Toronto Press, 1951. 
l'imprimerie. La reproduction sous forme d'imprimé - et de fichier électronique - de ce texte d'un auteur majeur devrait toutefois permettre de faire connaitre plus largement son manuscrit, en attendant la publication prochaine de son dernier opus ${ }^{13}$.

13. La publication d'une version abrégée des chap. IV, V et VI du manuscrit est prévue chez Rowman \& Littlefield sous le titre Harold Innis's History of Communications, vol. 1, William J. Buxton, Michael Cheney, and Paul Heyer (dir.). Un second volume, couvrant les matériaux des chapitres VII et VIII, est également en préparation. La rédaction remercie ici les éditeurs pour l'aimable autorisation à publier cet extrait. 
